\title{
Proton Radiography Studies for Proton CT
}

\author{
M. Petterson, N. Blumenkrantz, J. Feldt, J. Heimann, D. Lucia, A. Seiden, D. C. Williams, H. F.-W. Sadrozinski, \\ Member, IEEE, V. Bashkirov, R. Schulte, M. Bruzzi, D. Menichelli, M. Scaringella, C. Talamonti, G. A. P. Cirrone, \\ G. Cuttone, D. Lo Presti, N. Randazzo, V. Sipala
}

\begin{abstract}
We report the results of a beam experiment to develop proton Computed Tomography (pCT). The set-up consists of telescopes of silicon strip detectors at the entrance and exit of a phantom to predict the path of the proton within the phantom and of a crystal calorimeter to measure the proton energy loss with high precision. The energy loss permits calculating the integrated proton stopping power along each proton path from which the electron density distribution can be reconstructed. We describe the $2 \mathrm{D}$-image reconstruction of a lowcontrast phantom, derive the relationship between contrast, pixel size, and dose, and study the spatial resolution achievable with this set-up.
\end{abstract}

\section{INTRODUCTION}

$\mathrm{P}_{\mathrm{F}}$ ROTON radiation therapy is one of the most precise forms of noninvasive image-guided cancer therapy. It is based on the well-defined range of protons in material, with low entrance dose, a dose maximum ("Bragg peak"), and a rapid distal dose fall-off, providing better sparing of healthy tissue and allowing higher tumor doses than conventional radiation therapy with photons. At present, one can not fully exploit the potentials of proton therapy because conversion of Hounsfield values measured with x-ray computed tomography (CT) to relative electron density values is not always accurate [1]. The resulting uncertainties can lead to range errors from several millimeters up to more than $1 \mathrm{~cm}$ depending on the anatomical region treated. Additional uncertainties exist with respect to the target position relative to normal tissues in the treatment room that could be minimized by using proton CT for image-guiding the proton beam.

The long-term goal of our project is to develop the capability to use proton $\mathrm{CT}(\mathrm{pCT})$ instead of x-ray $\mathrm{CT}$ to minimize these uncertainties from the current value of 3-10 $\mathrm{mm}$ to $1-3 \mathrm{~mm}$. Previous work reviewed in [2] and our own preliminary studies [3-9] indicate that proton CT based on tracking of individual protons traversing an object from many different directions and measuring their energy loss and scattering angle may yield accurate reconstructions of electron

Manuscript received November 26, 2006. This work was supported in part by a seed grant from the Loma Linda University School of Medicine.

M. Petterson, N. Blumenkrantz, J. Feldt, J. Heimann, D. Lucia, A. Seiden, D. C. Williams, and H. F.-W. Sadrozinski are with the Santa Cruz Institute of Particle Physics, University of California, Santa Cruz, CA95064, USA (telephone: (831) 459-4670, email: hartmut@scipp.ucsc.edu).

V. Bashkirov and R. Schulte are with the Department of Radiation Medicine, Loma Linda University Medical Center, Loma Linda, CA 92354, USA (telephone: (909) 553-4243, e-mail: rschulte@dominion.llumc.edu).

M. Bruzzi, D. Menichelli, M.Scaringella, and C. Talamonti are with INFN and Università di Firenze, Dipartimento di Energetica, Via S. Marta 3, 50139 Firenze, Italy (telephone: +39-55-4796350, e-mail: bruzzim @ fi.infn.it).

G. A. P. Cirrone, G. Cuttone, D. Lo Presti, N. Randazzo, and V. Sipala are with the INFN, Laboratori Nazionali del Sud, Via S. Sofia 64, 95123 Catania, Italy (telephone: +39-95-542258, email: cirrone@Ins.infn.it) density maps with good density and spatial resolution, despite the fundamental limitation of energy straggling and multiple Coulomb scattering (MCS).
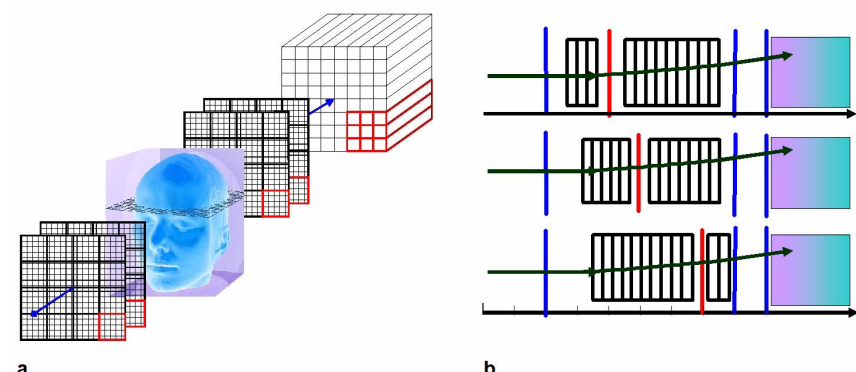

Fig. 1. a) Principle of the pCT system consisting of an entrance and exit telescope and a multi-array crystal calorimeter. A scaled-down prototype system is shown in red, while the full segmented system is shown in black. b) Sketch of the experimental beam set-up: the $200-\mathrm{MeV}$ proton beam enters from left, is tracked in the entrance silicon module (blue), passes through the segmented absorber (12 pieces of PMMA of $1.25 \mathrm{~cm}$ thickness each), and is tracked in the exit telescope (blue) before being stopped in the crystal. For the tracking studies, a "roving" silicon module (red) was placed at three positions within the absorber stack. For the calorimeter studies, both front and back of the phantom were equipped with a telescope, and a contrast resolution phantom was placed at the center of the PMMA stack.

\section{PCT: MEASUREMENT PRINCIPLE AND LIMITATIONS}

The goal to measure single protons leads to the following conceptual design of the pCT scanner, shown in Fig. 1 a: the proton locations and directions at the entrance and exit of the phantom/patient are measured each with a telescope consisting of two $x-y$ planes of silicon detectors. The energy is measured in a hodoscopic array of calorimeter crystals. Details are given in [8].

The challenge of proton-by-proton CT can be evaluated by a comparison with its established alternative, x-ray CT. A detection of individual protons requires a data acquisition system capable of recording particle rates in excess of $1 \mathrm{MHz}$. We have developed such a system for the readout of silicon strip detectors [9]. The curved trajectories of the protons inside the phantom create difficulties for the image reconstruction as well, and instead of a straightforward filtered back-projection algorithm, algebraic reconstruction techniques have been employed [10], [11].

Protons are undergoing multiple Coulomb scattering (MCS), which changes their direction and position depending on the amount of material traversed and the proton energy. The theory of MCS is well understood [12] and using it, the most likely path (MLP) inside the absorber can be reconstructed when the entrance and exit trajectories are 
measured external to the absorber. An analytical calculation of the MLP as a function of material, depth, displacement, and scattering angle has been derived in [6] and experimentally verified. The results of this experiment, described in more detail in [13], are reviewed briefly in the first part of this paper.

While x-ray CT uses a statistical evaluation of the absorption, pCT measures the energy loss of individual protons. In previous studies, we showed high contrast imaging using $140 \mathrm{MeV}$ protons [3]. The dose is proportional to the square of energy resolution (see below), thus mandating good energy determination for patient protection reasons. A recent beam test to measure the achievable contrast resolution and the implications for the dose and resolved voxel size is described in the second part of this paper.

\section{BEAM EXPERIMENT RESUlTS: TRACKING THE Most LIKELY PATH}

The theoretical MLP prediction and associated one and two sigma envelopes [6] use the well established Gaussian approximation of MCS theory [12]. The MLP depends both on lateral displacement and exit angle relative to the entry trajectory. For typical MLPs in homogeneous media the expected uncertainty is of the order of $300 \mu \mathrm{m}$. The objective of the tracking studies presented here and in more detail in [13] was to verify the theoretical predictions by tracking individual protons inside a segmented absorber.

A beam experiment with $200 \mathrm{MeV}$ protons was performed at the medical proton synchrotron located at Loma Linda University Medical Center (LLUMC). The protons were tracked with silicon strip detectors (SSDs) used before in the 1997 GLAST beam test [14]. In addition, a CsI calorimeter crystal provided energy measurement and a trigger for readout of the SSD system.

\section{A. Experimental Set-Up}

Details of the experimental set-up components and the data analysis are given in [13, [15], [16], and [17]. The set-up consisted of $\mathrm{x}-\mathrm{y}$ silicon modules used as entrance and exit telescopes, and a CsI calorimeter (Fig. 1b). The set-up was flexible in that it allowed for insertion of up to 12 absorber plates (1.25 cm PMMA each) and a "roving" silicon module between the telescopes. Data were taken both without absorber (for beam diagnosis), and with absorber to map out the MLP at different depths within the PMMA stack with the roving module. The three different locations of the roving module behind $3.75 \mathrm{~cm}, 6.25 \mathrm{~cm}$, and $12.5 \mathrm{~cm}$ PMMA are also shown in Fig. 1b. The beam-diagnostic configuration allowed measuring the entrance location and angle of the protons, i.e., the beam size and spread, while the set-up for evaluation of the MLP measured only the entrance location in one silicon module but not the entrance angle of the protons due to the limited number of available silicon modules. Since the MCS angle at the roving module location turned out to be much larger than the beam spread, this was an acceptable limitation.

\section{B. Most Likely Path as a Function of Exit Displacement and Angle}

The MLP analysis correlates the proton displacement in the roving module (i.e., lateral position relative to the entrance position) with the exit displacement and angle. The displacements in the roving module were determined for exitdisplacement bins of $\pm 200 \mu \mathrm{m}$ and exit-angle bins $\pm 5 \mathrm{mrad}$. Exit angles ranged from about $-80 \mathrm{mrad}$ to $+80 \mathrm{mrad}( \pm 4.5$ degrees). For comparison between the measured displacements and theory, the original MLP prediction for a uniform medium [6] was modified to include the air gaps of about $1.5 \mathrm{~cm}$ between the PMMA plates allowing for insertion of the silicon detectors. Fig. 2 shows the experimental and analytical MLP displacements in the roving modules at the three different depths within the absorber for four selected exit displacements and averaged over all exit angles. The MLP agrees with the data within less than $200 \mu \mathrm{m}$.

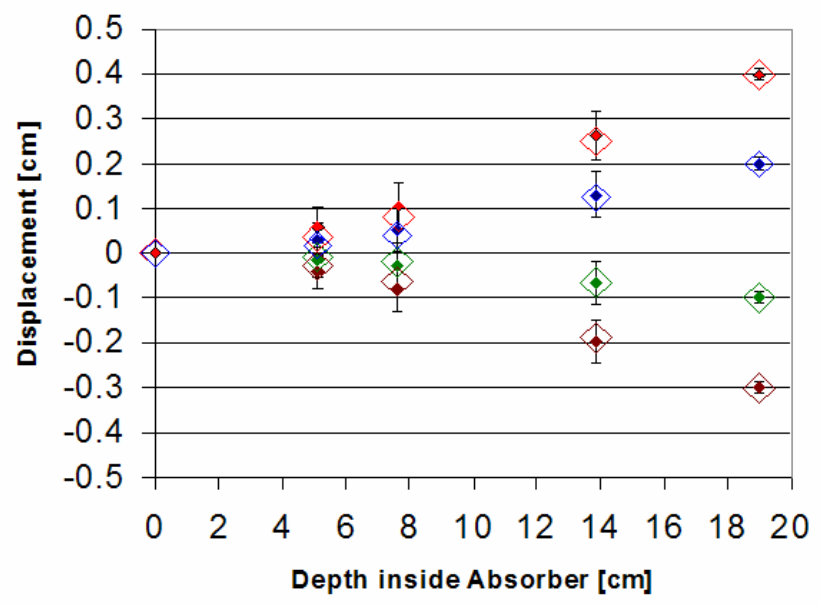

Fig. 2. Comparison of the measured displacement (mean \pm RMS) in the roving plane at 3 different absorber depths and for 4 different exit displacements (shown at $19 \mathrm{~cm}$ depth) with the analytically predicted MLP displacement (open symbols: the size of the symbol approximates the predicted MLP spread).

\section{Spread of the Displacement Distributions within the Absorber}

The spread of the displacements in the roving module for a given exit displacement of $-0.4 \mathrm{~cm}$ are shown in Table I. Three factors influence the observed spread in the roving modules. As mentioned before, the finite beam spread influences the spread of the experimental distributions. This will be simulated with Monte Carlo. Another factor is the bin size of the exit displacement, and a third uncertainty is introduced by the finite bin size of the exit angle.

As predicted by theory, the experimental spreads at a given roving location are independent of the exit displacement. A marked reduction of the spread of the displacement in the roving module was observed when the data were binned according to exit angle (central column in Table I). The use of the exit angle reduced the spread in the roving position by about 30 to $70 \mu \mathrm{m}$, depending on the PMMA depth. Dividing the data into angle bins thus reduces the experimental RMS by $10-20 \%$.

TABLE I 
SPREAD OF THE DISPLACEMENT DISTRIBUTIONS VS. DEPTH

\begin{tabular}{|cccc|}
\hline$z[c m]$ & $\begin{array}{c}\text { Exp } \\
\text { No angle } \\
\text { correction }\end{array}$ & $\begin{array}{c}\text { Exp } \\
\text { with angle } \\
\text { correction }\end{array}$ & $\begin{array}{c}\text { Theor. } \\
\text { MLP }\end{array}$ \\
\hline 5 & 370 & 340 & 250 \\
\hline 7.5 & 450 & 400 & 280 \\
\hline 14 & 480 & 410 & 330 \\
\hline
\end{tabular}

\section{Agreement between data and the MLP calculation}

The agreement between the data and the MLP calculation (modified for the finite air gaps at the position of the roving modules) has been tested further for various exit displacements and angles covering about $80 \%$ of the data. The data were binned into $\pm 200 \mu \mathrm{m}$ intervals in exit displacement and $\pm 5 \mathrm{mrad}$ intervals in exit angle. We found agreement between the experimental displacement and the MLP prediction within about $350 \mu \mathrm{m}$, with increasing disagreement at larger exit angles. We have started a Monte Carlo (MC) study of the experiment and a comparison between the MLP calculation and the complete MC results including the beam characteristics and the resolution of the detectors. Initial results [18] indicated good agreement at small angles, and the study is now extended to larger angles.

This beam test proved that, already at this stage, the location of the proton within a homogenous phantom can be predicted to better than $0.5 \mathrm{~mm}$, validating the MLP approach that uses only external track information for the prediction of the internal trajectories inside the phantom.

\section{CALORIMETER STUDIES}

Since the integrated stopping power is determined from the energy loss, understanding the calorimeter response in a $\mathrm{pCT}$ set-up is very important. A second dedicated test beam run was performed at LLUMC with essentially the same set-up as shown in Fig. 1b, but with the $6^{\text {th }}$ plate replaced by a PMMA plate with holes of different diameter and depth (Fig. 5 a), serving as "low-contrast resolution phantom". In this case the both front and back of the PMMA stack were provided with a tracking telescope. Details of the analysis are given in [19].

\section{A. Calorimeter response}

The light signal produced by the CsI crystal was read out with a photodiode (PD) into a charge-to-time-converter (CTC). The CTC signal was integrated into the readout chain permitting acquisition of calorimeter data and SSD data with a common FPGA [15].

At first, the calorimeter response was determined without PMMA absorbers for proton beam energies of $200 \mathrm{MeV}$ and $100 \mathrm{MeV}$, and during pedestal runs without beam (Fig. 3). Because of the low-energy tails in the energy distribution, only the high-energy part of the spectra was fit to a Gaussian, yielding the mean energy and the standard deviation (RMS). The calibration of the calorimeter yielded an almost linear relationship between CTC output and energy of the form

$$
C T C \#=.0002 E^{2}+2.5273 E+384.5
$$

Fig. 4 shows the energy resolution obtained at the two energies. The energy resolution after the protons traversed 11 or 12 planes of PMMA $(\sim 15 \mathrm{~cm})$ is also shown and is about 4 $\mathrm{MeV}$. This indicates that the energy straggling in the PMMA exceeds the energy resolution of the crystal calorimeter.
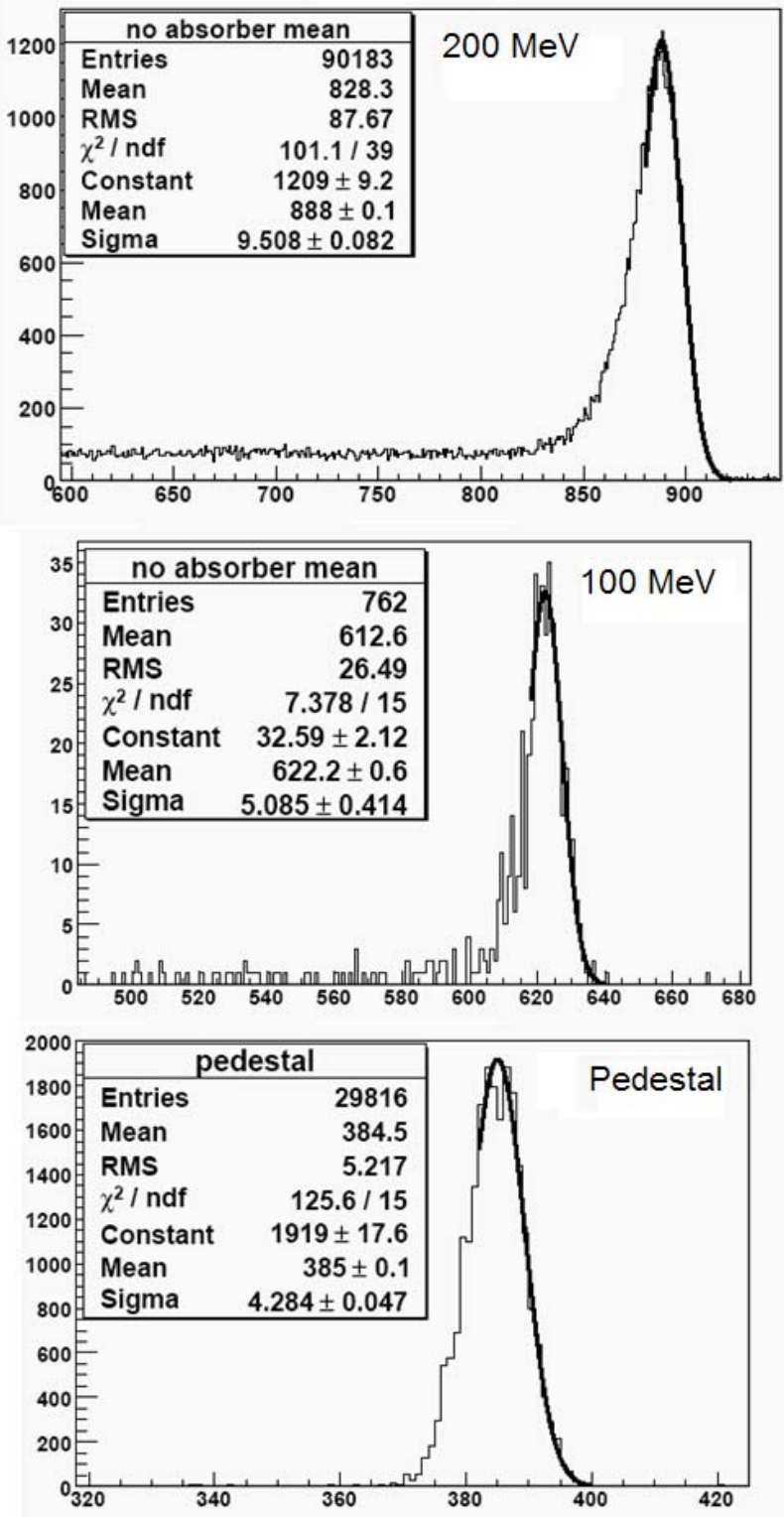

Fig. 3. Energy spectra (in CTC-number units) acquired by the CsI calorimeter for beam energies of $200 \mathrm{MeV}$ and $100 \mathrm{MeV}$, and without beam (pedestal). The parameters of the Gaussian fits, shown as solid lines, are in the inserts.

\section{B. Image Reconstruction}

Radiographic images of the density resolution phantom (Fig. 5a) were acquired with $200 \mathrm{MeV}$ protons and reconstructed by defining a $2 \mathrm{D}$ grid of pixels within in the SSDs. For each pixel, an energy spectrum similar to that of Fig. 3 was reconstructed. The fitted mean energy was then plotted on the grid resulting in the proton image of the phantom. 


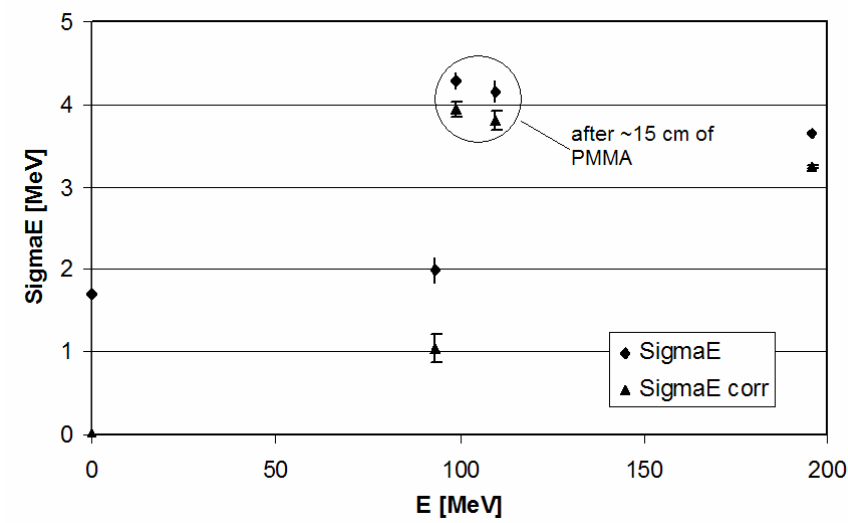

Fig. 4. Energy resolution (RMS) of the CsI calorimeter as a function of energy before and after correction for the pedestal are shown. The RMS after inclusion of $15 \mathrm{~cm}$ PMMA is also shown.

Images were reconstructed for pixel sizes of $1.2 \mathrm{~mm} \times 1.2$ $\mathrm{mm}, 5 \mathrm{~mm} \times 5 \mathrm{~mm}$, and $8 \mathrm{~mm} \times 8 \mathrm{~mm}$, respectively. Imaging results for $1.2 \mathrm{~mm}$ pixels are shown in Fig. 5b. The images of the $1.25 \mathrm{~cm}$ and the $0.6 \mathrm{~cm}$ holes in the phantom can be clearly seen on the background. For the analysis of the dosecontrast relationship, the areas inside the black boxes were chosen as the target and control voxels, respectively. The requirement of fitting the energy spectra had two consequences: First, if the number of protons in one pixel was lower than 10, a reliable fit could not be performed. Second, only about $1 / 4$ of all events in a spectrum contributed to the determination of the mean energy.

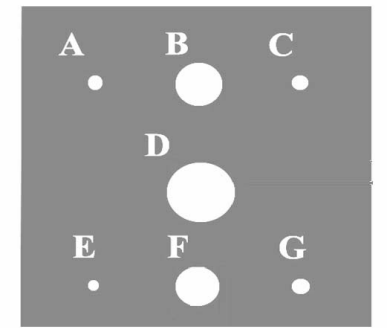

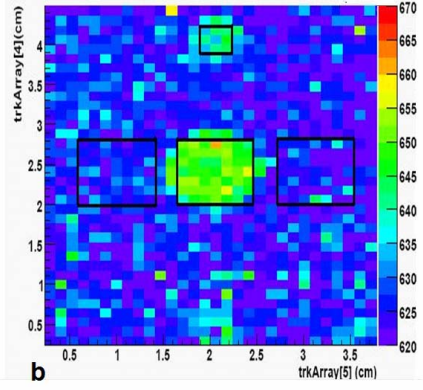
energy $\Delta E_{m} \propto \Delta \rho \cdot d$ and the standard error of the measurement $\sigma_{m}$ :

$$
S=\Delta E_{m} / \sigma_{m}
$$

For a Gaussian distribution, $\sigma_{m}=\sigma_{E} / \sqrt{N}$, where $N$ is the number of protons contributing to the energy measurement and $\sigma_{E}$ is the individual energy measurement uncertainty. For a difference of two or more standard deviations $(S \geq 2)$, the probability that this occurred due to noise is $p<0.05$. For a minimum of 2-sigma significance, $S=2$ :

$$
\Delta E_{m}=2 \sigma_{m}=2 \sigma_{E} / \sqrt{N}
$$

The absorbed dose $D$ at the voxel location is given by the deposited energy per unit mass and thus proportional to the local proton fluence $\boldsymbol{\Phi}=N_{p} /$ Area and stopping power:

$$
D \propto N_{p} / d^{2}
$$

As mentioned above, the number $N$ of protons contributing to the Gaussian fit was about $1 / 4$ of the total number of protons $N_{p}$ contributing to the dose. By combining equations (2)-(4) one obtains relationship between dose, voxel and pixel size $d$, energy resolution $\sigma_{E}$ and density variation $\Delta \rho$ :

$$
D \propto \frac{\sigma_{E}{ }^{2}}{\Delta^{2} \rho \cdot d^{3}}
$$

In Fig. 6 the required dose to resolve a density difference of $\Delta \rho=1.2 \mathrm{~g} / \mathrm{cm}^{3}$ with respect to background is shown for two different voxel sizes. The experimental relationship agrees well with what is expected from (5). In addition, the lower resolution limit from the requirement to have at least 10 protons for the fit of the energy spectrum is shown. These values can be used as input for the dose calculations in future image proton reconstruction studies with our set-up.

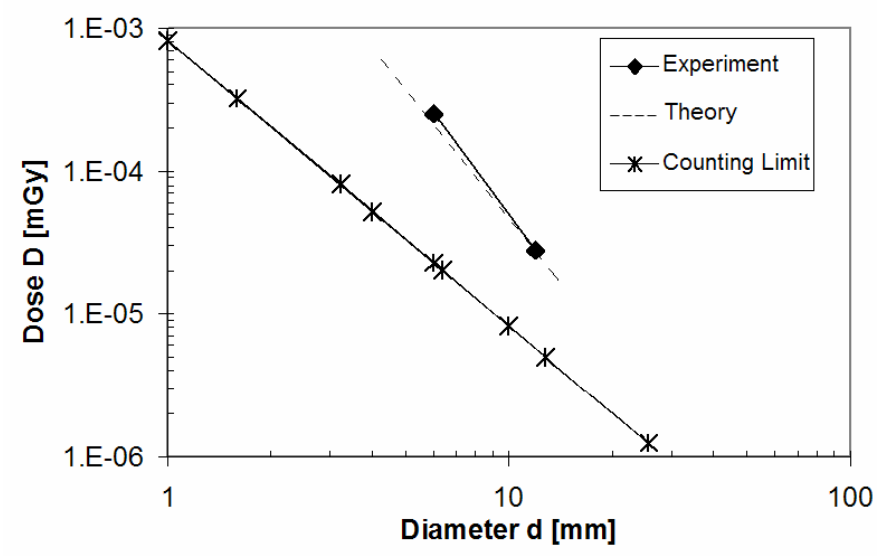

Fig. 6. Dose-voxel-size-contrast relationship for the experimental density increment $\Delta \rho=1.2 \mathrm{~g} / \mathrm{cm}^{3}$. The broken line indicates the power law of (5) matched to the lower experimental point. The line labeled "counting limit" is based on the fact that the energy determination required at least 10 protons.

\section{CONCLUSIONS}

We have measured the most likely path of $200 \mathrm{MeV}$ protons inside a segmented absorber of PMMA. The displacements of the protons from their original path agreed well with the theory of MCS. We showed that, as expected from the theory of the most likely path (MLP) [6], we can predict the 
trajectory of the proton inside a homogeneous absorber to better than $0.5 \mathrm{~mm}$.

We have imaged a low-contrast phantom inside a stack of PMMA plates by reconstructing the mean outgoing energy through fits of the energy spectra of each image pixel. For a reasonable density resolution, the images required a twosigma significance of the energy measurement. By systematically reducing the proton fluence within the data, we estimated the minimum dose level for resolving a density variation of $1.2 \mathrm{~g} / \mathrm{cm}^{3}$ for voxel sizes of $10 \mathrm{~mm}$ and $6 \mathrm{~mm}$. In addition, a minimum dose level for any contrast was derived based on the minimum number of protons required to fit the energy spectrum.

\section{ACKNOWLEDGMENT}

We thank the LLUMC accelerator staff for the smooth operation of the LLUMC synchrotron.

\section{REFERENCES}

[1] B. Schaffner and E. Pedroni, "The precision of proton range calculations in proton radiotherapy treatment planning: experimental verification of the relation between CT-HU and proton stopping power", Phys Med Biol. 43(6):1579-1592, 1998.

[2] H. F.-W. Sadrozinski, V. Bashkirov, B. Keeney, L. R. Johnson, S. G. Peggs, G. Ross, T. Satogata, R. W. M. Schulte, A. Seiden, K. Shanazi, and D. C. Williams, "Toward proton computed tomography", IEEE Trans. Nucl. Sci, vol 51, no.1, pp. 3-10, Feb. 2004.

[3] L. R. Johnson, B. Keeney, G. Ross, H. F.-W. Sadrozinski, V. Bashkirov, R. W. Schulte, K. Shahnazi. "Initial studies on proton computed tomography using a silicon strip detector telescope", Nucl Instr Meth A 514 (2003) 215.

[4] H. F-W Sadrozinski, M. Bruzzi, L. R. Johnson, B. Keeney, G. Ross, A. Seiden, D. C. Williams, L. Zhang, V. Bashkirov, R. W. Schulte, K. Shahnazi. "Issues in proton computed tomography", Nucl Instr Meth A 511: (2003) 275-281.

[5] L. R. Johnson, B. Keeney, G. Ross, H. F.-W. Sadrozinski, A. Seiden, D. C. Williams, L. Zhang,V. Bashkirov, R. W. Schulte, K. Shahnazi, "Monte Carlo studies on proton computed tomography using a silicon strip detector telescope". SCIPP 02/35.

[6] D C Williams, "The most likely path of an energetic charged particle through a uniform medium", Phys. Med. Biol. 49 2899-2911, 2004.

[7] R. W. Schulte, V. Bashkirov, M. C. Klock, T. Li, A. J. Wroe, I. Evseev, D. C. Williams, T. Satogata, "Density resolution of proton computed tomography", Med Phys. 2005, 32:1035-46.

[8] R. W. Schulte, V. Bashkirov, T. Li, Z. Liang, K. Mueller, J. Heimann, L. R. Johnson, B. Keeney, H. F.-W. Sadrozinski, A. Seiden, D. C. Williams, L. Zhang, Z. Li, S. Peggs, T. Saratoga, C, Woody, "Conceptual design of a proton computed tomography system for applications in proton radiation therapy", IEEE Trans. Nucl. Sci., vol 51, no.3, pp 866-875, June 2004.

[9] H. F.-W. Sadrozinski, V. Bashkirov, M. Bruzzi, M. Ebrahimi, J. Feldt, J. Heimann, B. Keeney, F. Martinez-McKinney, D. Menichelli, G.Nelson, G. Nesom, R. W. M. Schulte, A. Seiden, E. Spencer, J. Wray, and L. Zhang, "The particle tracking silicon microscope PTSM", IEEE Trans. Nucl. Sci., vol 51, no.5, pp 2032 - 2037, Oct. 2004.

[10] T. Li, Z. Liang, et al. " Reconstruction with most likely trajectory for proton computed tomography", SPIE Medical Imaging, 2004.

[11] T. Li Z. Liang J. V. Singanallur T. J. Satogata D. C. Williams, and R.W. Schulte, "Reconstruction for proton computed tomography by tracing proton trajectories: a Monte Carlo study", Med Phys. 33, pp 699-706, Mar. 2006.

[12] Yao WM et al., "Review of Particle Phyiscs, Chapter 27 Passage of Particles through Matter" J. Phys. G: Nucl. Part. Phys., vol. 33, pp 265267, Jul. 2006.

[13] N. Blumenkrantz, F. Feldt, J. Heimann, D. Lucia, H. F.-W. Sadrozinski, A. Seiden, W. Sowerwine, D. C. Williams, V. Bashkirov, R. Schulte, M. Bruzzi, D. Menichelli, M. Scaringella, G.A.P. Cirrone, G. Cuttone, N.
Randazzo, V. Sipala, D. Lo Presti, D. "Prototype Tracking Studies for Proton CT", IEEE NSS Conference Record 2005, vol. 1, pp 59 - 63, to be published in IEEE Trans. Nucl. Sci.

[14] W. B. Atwood, S. Ritz, P.Anthony, R.P.Johnson, W.Kroeger, H.F.W.Sadrozinski et al., "Beam test of Gamma-ray Large Area Space Telescope components", Nucl.Instrum.Meth. A446 (2000) 444-460.

[15] J. Heimann, "Developing an FPGA-based Readout for the pCT Detector System", Senior Thesis, UC Santa Cruz Physics Dept., June 2005.

[16] J. Feldt, "Preliminary Tracking Studies for Proton CT", Masters Thesis, UC Santa Cruz Physics Dept., December 2005.

[17] N. Blumenkrantz, "Tracking Study of $200 \mathrm{MeV}$ Protons within a PMMA Phantom", Senior Thesis, UC Santa Cruz Physics Dept., March 2006

[18] G. A. P. Cirrone et $a l$, "Detailed Monte Carlo Investigation of a Proton Computed Tomography System", Poster J03-25, 2005 IEEE NSS-MIC Symposium in Puerto Rico, Oct. 2005.

[19] M. K. Petterson, "Calorimeter Studies for Proton pCT", Senior Thesis, UC Santa Cruz Physics Dept., June 2006. 